\title{
DYNAMIC MASS EXCHANGE IN DOUBLY DEGENERATE BINARIES. II. THE IMPORTANCE OF NUCLEAR ENERGY RELEASE
}

\author{
W. Benz and A.G.W. Cameron \\ Harvard-Smithsonian Center for Astrophysics \\ 60 Garden Street, Cambridge, MA 02138 \\ and \\ R. L. Bowers \\ Applied Theoretical Physics, X-10, MS B259 \\ Los Alamos National Laboratory, Los Alamos, N.M. 87545
}

\section{INTRODUCTION}

The hypothesis that Type I supernova explosions originate in binary stars, in which the secondary overflows its Roche lobe and causes a white dwarf primary to pass a critical threshold for dynamical collapse, has gained in popularity over the last decade and a half (Truran and Cameron 1971; Whelan and Iben 1973; Wheeler 1982). During this period attention has also focussed on double-degenerate binary precursors of such systems, in which gravitational radiation of orbital angular momentum causes two white dwarf stars to spiral slowly together until the less massive of the stars begins to overflow its Roche lobe (Tutukov and Yungelson 1979; Webbink 1979; Iben and Tutukov 1984; Paczyński 1985). Cameron and Iben (1986) discussed the stability of such systems as the threshold of Roche lobe overflow was approached and for low mass ratios in which gravitational radiation can maintain a low rate of such overflow. Benz, Bowers, Cameron, and Press (1988, hereafter BBCP) computed the actual merging of a binary system composed of a $1.2 \mathrm{M}_{\odot}$ primary and a $0.9 \mathrm{M}_{\odot}$ secondary using a fully three-dimensional SPH code. Their simulation did not include the effect of nuclear energy release despite the fact that the temperature in the accretion shock reached the carbon ignition threshold, and therefore their conclusions about the structure of the merged object remained uncertain.

In this paper we present preliminary results for the merging of the same binary system as in $\mathrm{BBCP}$, but we have included in the code a reduced nuclear reaction network and we have allowed for radiation pressure effects. The binary stars were, as in BBCP, assumed to be two zero temperature carbon-oxygen white dwarfs. Since BBCP describes in sufficient detail the equations solved and the numerical method, we will only concentrate here on the new physics that has been introduced into the code.

\section{HYDRODYNAMIC EQUATIONS AND NUCLEAR REACTION NETWORK}

The results reported below were obtained using a fully three-dimensional hydrodynamic code based on the SPH scheme, which includes self-gravity and which makes no assumptions whatsoever about the flow symmetry. The hydrodynamics is described by the momentum equation in its Lagrangian form:

$$
\frac{d \mathbf{v}}{d t}=-\frac{\nabla \mathbf{P}_{\text {tot }}}{\rho}-\nabla \phi+\mathbf{S}_{\mathbf{v i s c}}
$$

where $\mathbf{S}_{\text {vise }}$ is the classical artificial viscous stress which is introduced to model kinetic energy 
dissipation in shocks; and the gravitational potential $\phi$ is obtained from Poisson's equation

$$
\nabla^{2} \phi=4 \pi G \rho
$$

The mass density is denoted by $\rho$, the gravitational constant by $G$, and $\mathbf{v}$ is the velocity of a mass element.

In $\mathrm{BBCP}$ the total fluid pressure did not include radiation effects; we now add this term by writing the total pressure to be equal to the sum of a degenerate electron pressure, a nondegenerate gas pressure, and a radiation pressure term:

$$
P_{\text {tot }}=P_{\text {deg }}+P_{\text {gas }}+P_{\text {rad }}
$$

In the degenerate regime, the pressure is obtained, as in BBCP, from the ideal, fully relativistic equation of state (Chandrasekhar 1939). The gas pressure is found from the perfect gas equation of state. The radiation pressure term has the usual form:

$$
P_{\mathrm{rad}}=\frac{1}{3} a T^{4}
$$

In order to compute the gas and radiation pressure terms we first have to know the temperature. To obtain the temperature we solve for each particle the equation:

$$
U_{\text {non-deg }}=U_{\text {rad }}+U_{\text {therm }}=a T^{4}+c_{\mathrm{v}} T
$$

where $c_{\mathbf{v}}$ is the specific heat at constant volume and $U_{\text {non-deg }}$ is the non-degenerate part of the total internal energy which is obtained by subtracting the degenerate energy from the total internal energy. The degenerate energy is only a function of density and can be readily obtained once $\rho$ is known, and the total internal energy is given by solving the energy conservation equation.

As mentioned above, the high temperature reached in the accretion shock indicates that nuclear energy release should be included in the code. Of course, a full network coupled with the 3D hydro code would be impractical because of the computer time and storage that would be required. We therefore used the same reduced network as in Benz, Hills, and Thielemann (1988, hereafter BHT). This network, developed for the study of white dwarf collisions, was devised having two simplifications in mind. First, since the dynamics has to be modelled but not the detailed chemical composition, the network should only serve the purpose of reproducing the energy generation accurately. This energy release should also take place over the correct timescale. Second, the bulk composition of white dwarfs, generally a mixture of carbon and oxygen, with perhaps some helium, implies that "multiple alpha-particle" nuclei will dominate and that neutron- and proton-induced reactions can be neglected in a first approximation. For fast nuclear burning in which the principal products produced are not heavier than magnesium or silicon, as turned out to be the case here, a simple chain of these multiple alpha-particle nuclei, emitting and absorbing alpha-particles, gives an acceptable approximation to a larger network, and that is what was used here.

Further details about the equations, the nuclear reaction network, and the numerical method can be found in BBCP and in BHT.

\section{RESULTS}

Figure 1 shows a selected number of snapshots of the binary dissolution. The velocity vectors are plotted projected onto the orbital plane. The magnitude of the minimum and maximum 
velocity vectors is indicated on the second line above each frame in units of $6363 \mathrm{~km} / \mathrm{s}$. Time is given on the upper line in units of 1.10 seconds and each frame is $5.6 \times 10^{9} \mathrm{~cm}$ on a side. The initial conditions were taken identical to those in BBCP, that is two initially non-rotating, zero temperature white dwarfs. The simulation begins with the secondary filling its Roche lobe. We further assume that angular momentum losses due to gravitational radiation can be neglected during the actual merging.

After a few seconds, mass transfer starts and very quickly grows to reach values as high as $0.0525 \mathrm{M}_{\odot} / \mathrm{s}$. As expected, the dissolution of the binary proceeds in a very similar way to that reported in $\mathrm{BBCP}$, and the secondary is again completely destroyed in about 60 seconds, i.e., three original orbital periods. We pointed out in $\mathrm{BBCP}$ that this extremely rapid merging is due to the fact that not only mass but a substantial amount of angular momentum is transferred into the disk orbiting the primary which results in the two stars moving closer rather than away from each other.

The resulting merged object, although more massive than a Chandrasekhar mass, does not collapse for the same reasons as found in BBCP. The inner part of the material added to the primary is no longer degenerate but is supported by thermal pressure, whereas the outer parts are supported by rotation. It will take at least one cooling time for the potential supernova event to take place.

As predicted, we find that the temperature rises high enough in the accretion shock to ignite carbon burning. However, the density and temperature only reach values for which carbon burns very slowly, and the amount of nuclear energy released only marginally affects the dynamics. The major effect of nuclear burning is to allow considerably more mass loss from the system. Although still a small amount, the mass lost, now $0.051 \mathrm{M}_{\odot}$, is about 9 times larger than was found in $\mathrm{BBCP}$. Most of the ejected mass has been highly processed. ${ }^{12} \mathrm{C}$ has been reduced in abundance from 0.5 to 0.164 , and ${ }^{16} \mathrm{O}$ at 0.5013 is slightly above the original abundance of 0.5 . The major elements that have been produced are ${ }^{24} \mathrm{Mg}(0.184),{ }^{28} \mathrm{Si}(0.138)$, and ${ }^{32} \mathrm{~S}(0.012)$. The equivalent mass of the processed material ejected into the interstellar medium prior to the eventual explosion amounts to $0.0094 \mathrm{M}_{\odot}$ of ${ }^{24} \mathrm{Mg}$ and $0.0071 \mathrm{M}_{\odot}$ of ${ }^{28} \mathrm{Si}$. This should not be enough to produce any visible signature later on when the ejecta from the supernova explosion runs into this earlier debris.

The chemical composition of the merged object is practically unchanged from its original value. Processed elements are only present in traces. The most abundant of them, again ${ }^{24} \mathrm{Mg}$ and ${ }^{28} \mathrm{Si}$, have abundances of 0.0073 and 0.0054 , respectively. All these processed elements are located in the hot envelope surrounding the original primary. Practically no burning took place involving material from the primary. The merging process will therefore leave no obvious signature, at least as far as the chemical composition of the supernova progenitor is concerned.

This work has been supported in part by NASA grant NGR 22-007-272. One of us (W.B.) also aknowledges partial support from the Swiss National Science Foundation and would like to express his gratitude to the Theoretical Astrophysics Group of the Los Alamos National Laboratory for their hospitality.

\section{REFERENCES}

Benz, W., Bowers, R.L., Cameron, A.G.W, and Press, W.H., Astrophys. J., submitted. Benz, W., Hills, J.G., and Thielemann F.K., Astrophys. J., in preparation.

Cameron, A. G. W., and Iben, I., Jr., Astrophys. J., 305, 228 (1986).

Chandrasekhar, S., Introduction to the Study of Stellar Structure, University of Chicago Press, 
Chicago, Illinois, Chap. XI (1939).

Chandrasekhar, S., Classical Ellipsoidal Figures of Equilibrium, Yale University Press (1969). Iben, I., Jr., The Galaxy, Ed. G. Gilmore, V. Carswell (Dordrecht: Reidel), p. 365 (1987).

Iben, I., Jr., Astrophys. J., 324, 355 (1988).

Iben, I., Jr., and Tutukov, A. V., Astrophys. J. Suppl., 54, 535 (1984).

Iben, I., Jr., and Webbink, R. F., in proceedings of the "second conference on faint blue stars",

Ed. A. G. Davis-Philip, D. F. Hayes, J. W. Liebert (L. Davis Press), p. 401 (1988).

Paczyński, B., in Cataclysmic Variables and Low-Mass $X$-Ray Binaries, Ed., D. Q. Lamb and J.

Patterson (Dordrecht: Reidel), p. 1 (1985).

Truran, J. W., and Cameron, A. G. W., Ap. Space Sci., 14179 (1971).

Tutukov, A. V., and Yungelson, L. R., Acta Astr., 23, 665 (1979).

Webbink, R. F., in White Dwarfs and Variable Degenerate Stars, Ed. H. M. Van Horn and V.

Weidemann (New York: University of Rochester Press), p. 426 (1979).

Webbink, R. F., Iben, I., Jr., in proceedings of the "second conference on faint blue stars", Ed.

A. G. Davis-Philip, D. F. Hayes, G. W. Liebert, (L. Davis Press), p. 445 (1988).

Wheeler, J. C., in Supernovae: A Survey of Current Research, Ed. M. J. Rees and R. J. Stoneham

(Dordrecht: Reidel) p. 167 (1982).

Whelan, J. C., and Iben, I., Jr., Astrophys. J., 186, 1007 (1973).

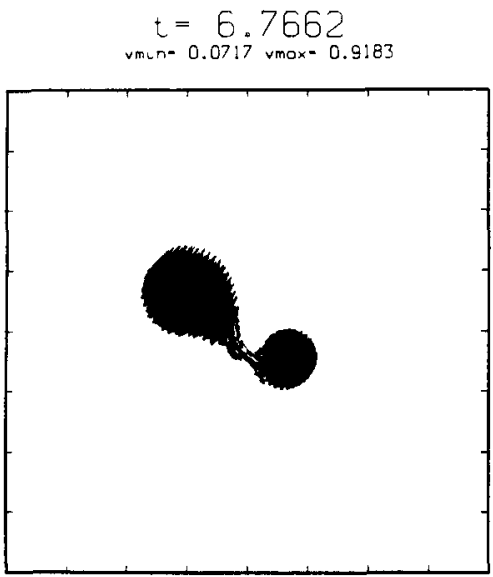

$t=25.9820$

vmin= 0.0076 vmax -0.9988

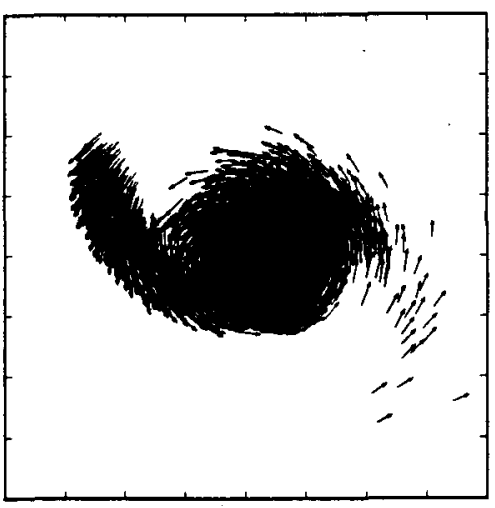

$t=17.6652$

vmen- 0.0229 vmax-1.0977

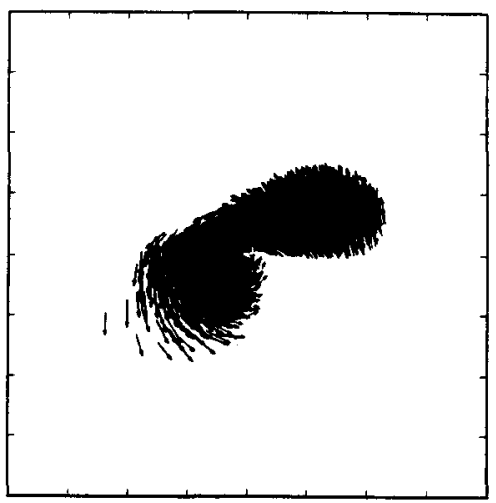

$t=38.7989$

vmเn= 0.0118 vmox= 0.9240

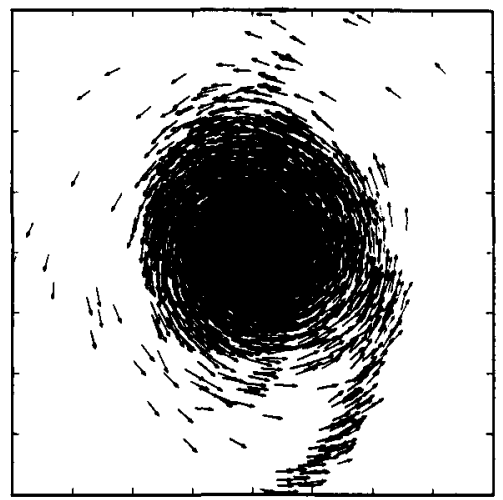

